\title{
A model involving meteorological factors for short- to-medium term water level prediction of small- and medium-sized urban rivers
}

\section{Yawei Qin}

Huazhong University of Science and Technology

\section{Yongjin Lei}

Huazhong University of Science and Technology

\section{Xiangyu Gong}

Huazhong University of Science and Technology

Wanglai Ju (D954814963@qq.com )

Huazhong University of Science and Technology https://orcid.org/0000-0002-2446-9677

\section{Research Article}

Keywords: Water level prediction, Meteorological data, Urban area, Short-to-medium term prediction, Particle swarm optimization, Support vector machine

Posted Date: June 7th, 2021

DOl: https://doi.org/10.21203/rs.3.rs-298449/v1

License: (c) (i) This work is licensed under a Creative Commons Attribution 4.0 International License. Read Full License

Version of Record: A version of this preprint was published at Natural Hazards on October 19th, 2021. See the published version at https://doi.org/10.1007/s11069-021-05076-y. 
Title: A model involving meteorological factors for short-to-medium term water level prediction of small- and medium-sized urban rivers

\author{
Author: Yawei Qin ${ }^{1,2} \cdot$ Yongjin Lei $^{1 \cdot}$ Xiangyu Gong ${ }^{1} \cdot$ Wanglai Ju$^{1}$ \\ corresponding author: Wanglai Ju E-mali: 954814963@qq.com
}

\begin{abstract}
Affiliation:
1 School of Civil and Hydraulic Engineering, Huazhong University of Science and Technology, Wuhan 430074, China
\end{abstract}

2 Wuhan Huazhong Keda Civil Engineering Testing Center, Wuhan 430074, China

\begin{abstract}
With the increasing of extreme weathers, cities, especially the small- and medium-sized urban rivers with the protection areas less than 200 square hectares, are experiencing significantly more flood disasters worldwide. Heavy snowfalls and rainfalls can rapidly overflow these rivers and cause floods due to the their unique geographic locations and fast runoff and confluence. Therefore, it is particularly important to accurately predict the short-to-medium term water levels of such rivers for reducing and avoiding urban floods. In the present work, a particle swarm optimization (PSO)-support vector machine (SVM) water level predication model was constructed by combining PSO and SVM and trained with the meteorological data of Wuhan, China, and the water level data of Yangtze River. The PSO-SVM model is able to lower mean square error (MSE) 70.47\% and increase coefficient of determination $\left(R^{2}\right) 7.02 \%$ of the prediction results, as compared with SVM model alone. The highly accurate PSO-SVM model can be used to predict river water level real-time using the hourly weather and water level data, which thereby provides quantitative data support for urban flood control, construction management of water projects, improving response efficiency and reducing safety risks.
\end{abstract}

Keywords Water level prediction - Meteorological data $\cdot$ Urban area $\cdot$ Short-to-medium term prediction $\cdot$ Particle swarm optimization $\cdot$ Support vector machine 
2 Small- and medium-sized rivers in urban span multiple administrative areas and possess unique 3 characteristics of shallow riverbed, small cross-section, limited and incomplete hydrological information, 4 and connections with large areas of impervious layers of cities (Zhang et al. 2016), which makes them vulnerable to extreme weathers. Local heavy snowfalls and rainstorms can seriously impact the urban areas around a river if its flood discharge capacity is poor (Rao et al. 2019). In 2019, extreme weathers in the cities of India, China, the United States, Japan, and Europe caused the floods and other disasters of over 25 billion U.S. dollars damages. The United Nations has called for preventing extreme weather from threatening human life. Therefore, the short-to-medium term water level prediction of small and medium-sized urban rivers based on meteorological data are significantly important.

Water level prediction has been extensively studied worldwide, especially for large rivers due to availability of large amounts of hydrologic monitoring data. Efficient water level prediction models can be constructed using various mathematical models based on the historical water level data of such rivers for quantitative analyses. For example, Barrameda et al constructed a model by combing back propagation neural network (BPNN) and SVM to predict the rainfall and water level of the Calinog River in Iloilo City, Philippines which showed higher accuracy than SVM alone (Barrameda et al. 2018). Shiri et al. obtained more accurate prediction of the water level in Urmia Lake using extreme learning machine (ELM) than using genetic programming (GP) and artificial neural network (ANN) (Shiri et al. 2016). A short term water level prediction model combining genetic algorithm (GA) and neural network was established and successfully applied to 15 water level stations on four major rivers of South Korea, showing great application potentials for water level predictions at different stations under different conditions (Lee et al. 2013). Adaptive neural fuzzy inference system (ANFIS) and differential integrated moving average autoregressive (ARIMA) model were also successfully applied to the water level prediction of the Klang River in Malaysia (Galavi et al. 2013). Lin et al. combined a climate model with digital weather data to predict severe precipitation anomalies of a few days to a few months ago in the Yangtze River Basin, and discussed the important role of seasonal dynamic prediction in flood management of the Basin. (Lin et al. 2005). A comprehensive clustering, classification, and regression framework model was constructed for the real-time water level prediction of the Yellow River Basin with its high sediment composite considered (Zhao et al. 2019). Hin and Othman predicted the water level in Lakec, Malaysia using classification and data mining, specifically for the uneven rainfalls caused by the monsoon season (Hin and Othman 2020). Zhu et al. reported a model based on BP artificial neural network for the flood season water level prediction of the Pearl River Basin (Zhu et al. 2005). Xie et al. also developed a BP artificial neural network-based model for the water level prediction on the Yangtze River hydrological station. They used the temporal differences of water level and flow as the input and output of the BP network model to improve the prediction accuracy during the flood season (XIE et al. 2005). Yadav and Eliza proposed the daily water level prediction using a mixed wavelet support vector machine model with the daily lake water level and hydrometeorological data of Lake Loktaq as the inputs (Yadav and Eliza 2017).

To sum up, a foundation has been established for the quantitative water level prediction of large rivers. Small- and medium-sized rivers, especially the small- and medium-sized urban rivers that are more 
sensitive to meteorological data and are susceptible to the mainstream and adjacent tributaries, are rarely involved. The studies of tributaries are less systematic and there are fewer hydrological and weather data for the tributary streams, as compared with mainstreams. Therefore, it is difficult to obtain accurate and comprehensive information for the quantitative analysis. In view of this, we have constructed a PSO and SVM algorithms based model for the short-to-medium term water level prediction of small- and mediumsized rivers in complex urban areas using meteorological data and water level of mainstream as the variables, aiming to explore the water level predictions of small- and medium-sized urban rivers in complex environments.

\section{Influencing factors}

Compared with large mainstream rivers, small- and medium-sized urban rivers are more sensitive to short- and medium-term weather changes because of their small catchment area (Simon et al. 2018). Their water changes are every random due to the complex surrounding environments, which makes their water level prediction very challenging.

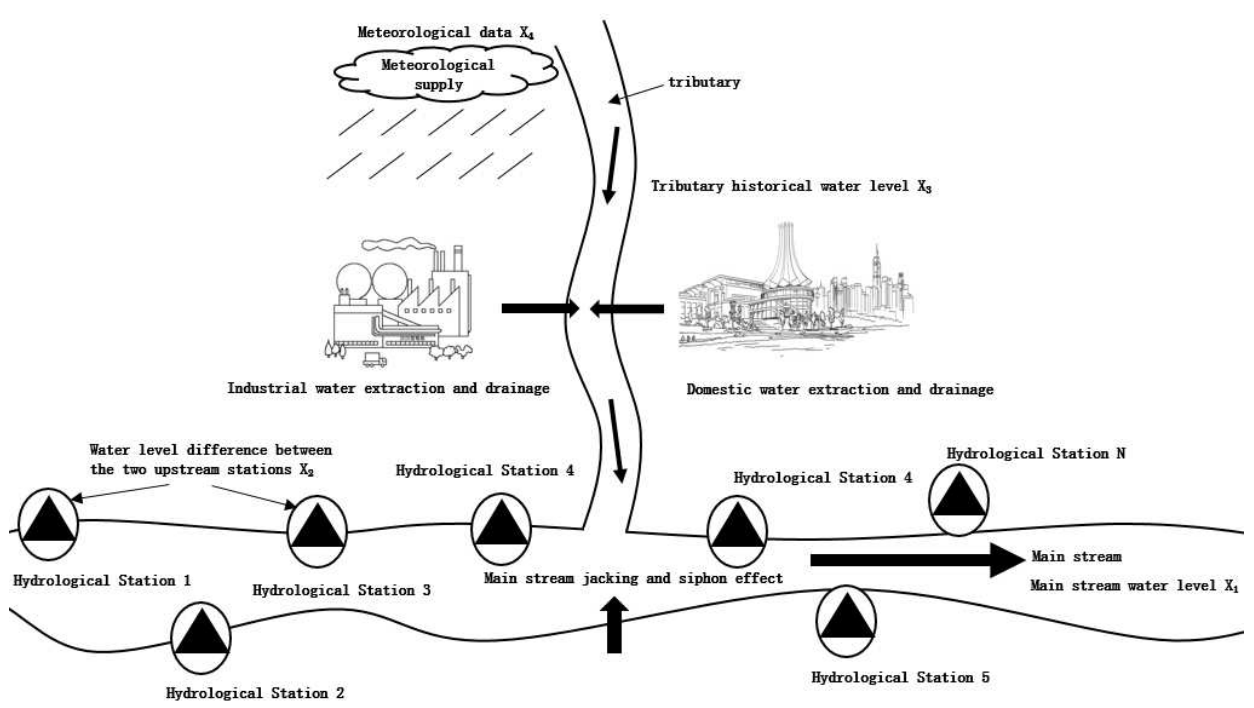

Fig. 1 Factors affecting water level

Fig. 1 shows the factors affecting the water level of the small- and medium-sized urban river. As can be seen, meteorological supply, industrial and domestic water extraction and drainage, and the water levels of mainstream are the main factors. Historical data suggest that the mainstream water level $\left(\mathrm{X}_{1}\right)$ imposes jacking effects on the tributary water level during the wet season when it is higher than the water level of the tributary (YAO et al. 2018), and shows siphon effects during the dry season when it water level is lower. The influencing variable $\mathrm{X}_{2}$ is used to eliminate the spatial variability of the mainstream water. Therefore, the difference between the water levels of two hydrological stations on the mainstream closest to the tributary is selected as $X_{2}$ (Nkiaka et al. 2018). Influencing variable $X_{3}$ represents the historical water level data of the tributary. Influencing variable $\mathrm{X}_{4}$ reflects the impact of short- and medium-term meteorological supply on the water level of the tributary. Empirical evidence suggests that the industrial and domestic water drainage is mainly related to urban development and has been in a stable state. It has 

shown no obvious nonlinear effect on the tributary water level, and thus is excluded from the model. The final selected influencing variables are listed in Table 1.

Table 1 Influencing variables for predictive modeling

\section{Prediction model theory}

The water level change with the influencing variables $\mathrm{X}_{1}, \mathrm{X}_{2}, \mathrm{X}_{3}$, and $\mathrm{X}_{4}$ is a highly uncertain nonlinear dynamic process in complex environments. Therefore, a suitable mathematical model is extremely important for predicting such multivariate chaotic sequence. At present, linear regression model (Kühn and Schöne 2017), artificial neural network model (Adamowski and Chan 2011; Khan and Coulibaly 2006; Kia et al. 2012; Yarar et al. 2009), VOLTERRA series adaptive model (Qiao et al. 2020) and SVM model (Kisi et al. 2015; Wang et al. 2010; Wei 2012) are the major mathematical models used for the prediction of multivariable chaotic sequences. Among them, the SVM model based on statistical learning theory and structural risk minimization principle has demonstrated great advantages for solving the nonlinear regression problems of small samples, such as clear theoretical foundation, global optimization and strong generalization ability. Yet the accuracy of the model is significantly affected by the parameter settings (Huang and Dun 2008). To make up for this shortcoming, the particle swarm optimization (PSO) with excellent global search capability and high efficiency can be used to optimize the parameters for SVM model. Therefore, we propose a PSO-SVM model to comprehensively predict the short-to medium term water levels of urban rivers.

\subsection{Particle swarm optimization (PSO)}

The particle swarm algorithm used for parameter optimization treats each individual as an particle in an n-dimensional search space, and each particle flies in this space at a certain speed (Selakov et al. 2014; Shrivastava et al. 2015). The position of each particle is a potential solution. The fitness is obtained from the objective function. The SVM model based on particle swarm algorithm updates its position and velocity according to the best position of the particle swarm and the best position of each particle, and gradually approaching the best position. The speed update and position update can be described as Eq. 1. 


$$
\left\{\begin{array}{l}
v_{i}^{k+1}=\omega v_{i}^{k}+c_{1} r_{1}\left(P_{\text {best }}-X_{i}^{k}\right)+c_{2} r_{2}\left(g_{\text {best }}-X_{i}^{k}\right) \\
X_{i}^{k=1}=X_{i}^{k}+v_{i}^{k+1}
\end{array}\right.
$$

102

103

104

105

106

107

108

109

110

111

112

113

114

115

116

117

118

119

120

121

122

123

124

125

126

where $v$ is the particle velocity, $\omega$ is the inertia weight, $c_{1}$ and $c_{2}$ are the acceleration factors, $g_{\text {best }}$ is the optimal position of each particle, $K$ is the number of iterations, $i$ is the population size, $X$ is the particle position, and $r_{1}$ and $r_{2}$ are the random numbers from [0,1]. The iteration is stopped as the preset maximum number of iterations is reached or the position obtained by PSO is higher than the preset minimum adaptive threshold.

\subsection{Support vector machine (SVM)}

SVM algorithm is a machine learning method based on statistical learning theory, which can effectively solve the nonlinear and high-dimensional recognition problems of small samples (Anirudh and Umes 2007; Eslamian et al. 2008; Moghaddamnia et al. 2009; Zakaria and Shabri 2012). SVM nonlinear regression prediction is based on structural risk minimization principle and Vapnik-Chervonenkis (VC) dimension theory. An optimal decision function can be constructed by nonlinear mapping and the linear regression is conducted in a high-dimensional space. The linear regression function can be expressed as Eq. 2.

$$
f(x)=\omega \cdot x+b
$$

where $\omega$ is the generalized parameter of the function.

The regression function is optimized with the $\varepsilon$-insensitive loss function, and the best function is determined with the minimum value of the function as shown in Eq. 3 and 4.

$$
\begin{gathered}
\min R\left(\omega, \xi, \xi^{*}\right)=\frac{1}{2} \omega^{2}+C \sum_{i=1}^{t}\left(\xi_{i}+\xi_{i}^{*}\right) \\
s . \mathrm{t}\left\{\begin{array}{l}
f\left(x_{i}\right)-y_{i} \leq \xi_{i}^{*}+\varepsilon, \\
f\left(x_{i}\right)-y_{i} \leq \xi_{i}+\varepsilon, i=1,2, \ldots, t \\
\xi_{i}, \xi_{i}^{*} \geq 0,
\end{array}\right.
\end{gathered}
$$

where $\xi_{i}$ and $\xi_{i}^{*}$ are the relaxation factors used to smooth the trend curve of the function and solve the calculation error of the regression, $C$ is a constant introducted to compromise the balance, and $\varepsilon$ is a constant for the error analysis.

The nonlinear regression function can be obtained by quadratic programming as Eq. 5 .

$$
f(x)=\sum_{i=1}^{n}\left(\beta_{i}-\beta_{i}^{*}\right) K\left(x_{g} x_{i}\right)+b
$$

where $K\left(x_{g} x_{i}\right)$ is the kernel function of SVM, and $\beta_{i}$ and $\beta_{i}^{*}$ are the Lagrangian multipliers. 
128 SVM can perform regression analysis alone, but its accuracy is significantly affected by the selection of its kernel function parameters for water level prediction. The cross-validation of kernel function itself usually falls into a local optimal solution, and thus cannot provide the global the optimal solution, causing low prediction accuracy of SVM. Herein, the parameters of SVM are iteratively optimized using particle swarm algorithm to build a desired model for the short-to-medium term water level prediction of smalland medium-sized urban rivers. Fig. 2 shows the flowchart of the PSO-SVM modeling process.

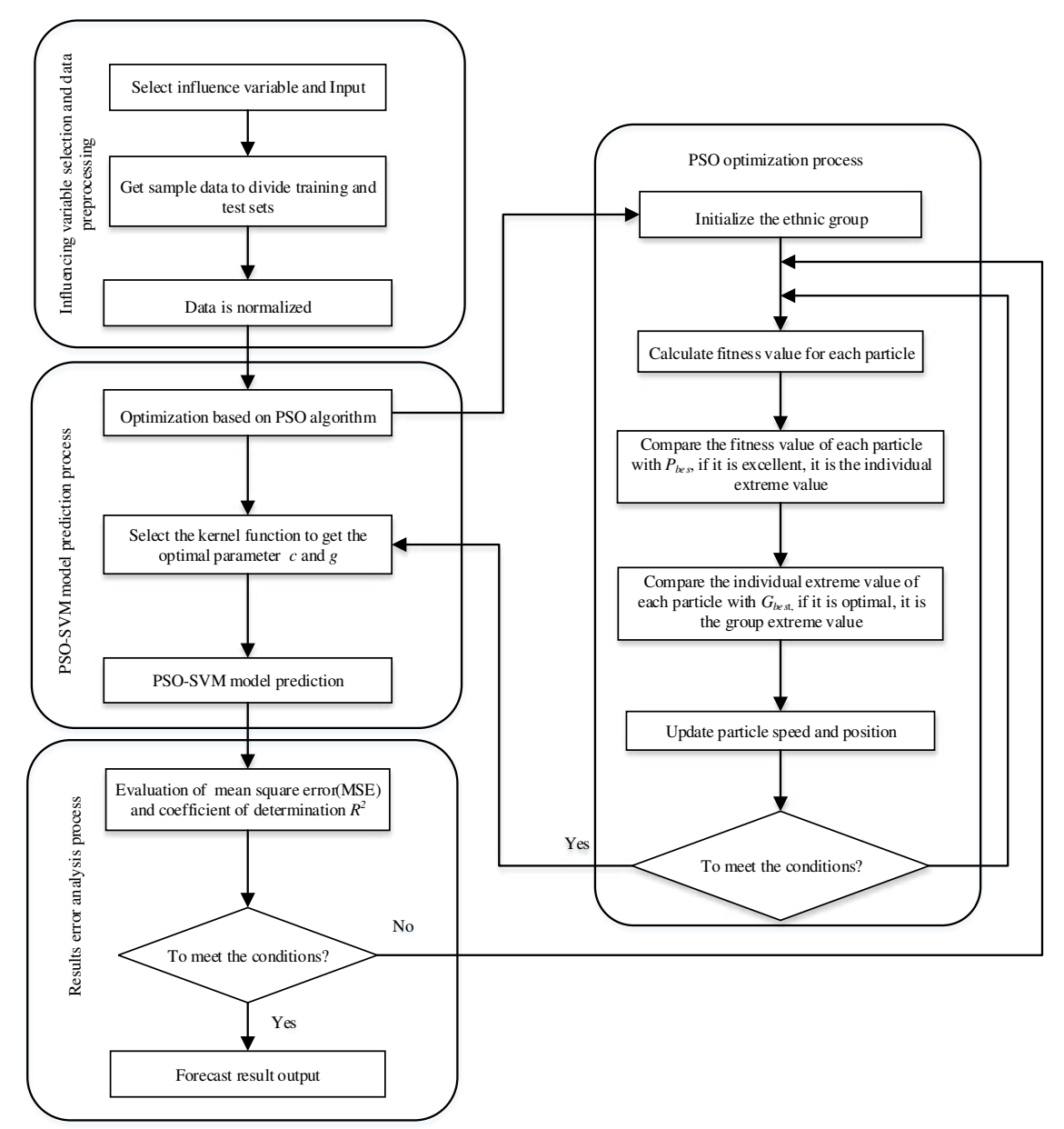

Fig. 2 Flowchart of PSO-SVM modeling for water level prediction .

136 The modeling process comprises the following steps:

137 Step1 Data acquisition and processing

138 The data of the factors that affect the water level can be classified as structured data and unstructured 139 data. The mainstream water level $\left(X_{1}\right)$, the upstream water level difference $\left(X_{2}\right)$, and the historical water 
level $\left(\mathrm{X}_{3}\right)$ are structured data, which can be accurately and immediately obtained from hydrological stations. Meteorological data are unstructured data. To more specifically characterize the weather changes, the original meteorological data are quantitatively processed by a scoring method based on the criteria of precipitation or not and the amount of precipitation. No rain, shower, light rain, moderate rain, heavy rain, and rainstorm are respectively scored as 0 point, 1 point, 2 points, 3 points, 4 points and 5 points (Caizhi and Xueyu 2003). To accelerate the parameter optimization, improve the model training efficiency, and reduce memory space, the sample data are normalized with Eq. 7.

$$
y=\frac{y_{\max }-y_{\min }}{x_{\max }-x_{\min }}\left(x-x_{\min }\right)+y_{\min }
$$

Step2 PSO and tuning

The accuracy and performance of SVM model are mainly affected by its penalty factor $c$ and kernel parameter $g$, and thus those two parametersare optimized using partice swarm algorithm.

Step3 Model training

According to the fitting principle of parameter optimization by PSO, the SVM model is trained with the parameter optimization results obtained by PSO in step 2 and the normalized data of step 1 . The optimal fitting function is obtained as Eq. 8.

$$
\bar{D}=f(y)=\left[\sum_{i=1}^{k}\left(a_{i}-a_{i}^{*}\right) \exp \left(-\left\|y-y_{k}\right\|^{2}\right) / 2 \delta^{2}\right]+b
$$

where $a_{i}$ and $a_{i}^{*}$ are the Lagrange factors corresponding to the SVM and $b$ is a bias term. This trained fitting equation is then used as a PSO-SVM model for water level prediction.

Step4 Results and error analysis

To more comprehensively evaluate the model, the prediction results are subjected to error analysis for goodness of fit, namely the mean square error (MSE), and coefficient of determination $\left(R^{2}\right)$. The closer to 0 the MSE is, the smaller the prediction error and the higher the accuracy of the model. The $R^{2}$ value closer to 1 suggests smaller prediction error and higher the prediction accuracy. The model is considered accurate if MSE is smaller than 0.1 and $R^{2}$ is greater than 0.9. Otherwise, the SVM parameters are further optimized by PSO and the prediction model is then rebuilt until the accuracy requirements are met.

\section{Shor- and medium-term water level prediction of Xinhe Bridge using PSO-SVM model}

\subsection{Hydrologic engineering background analysis}

Xinhe Bridge is over a small- to medium-sized tributary of Wuhan city, $\sim 350 \mathrm{~m}$ away from the junction of the Sheshui River estuary and the Yangtze River. It is in the subtropic climate zone with four distinct seasons and abundant rainfalls. According to the historical hydrological data provided by Wuhan meteorological observatory, the average annual precipitation of the city is about 125 days, and the 
rainfalls mainly occur in summer. Atmospheric precipitation, surface water and adjacent water systems, and drainage of urban and factory water are the main water supply sources. Fig. 3 shows the distribution of water systems and the locations of hydrological monitoring stations near the object.

174

175

176

177

178

179

180

181

182

183

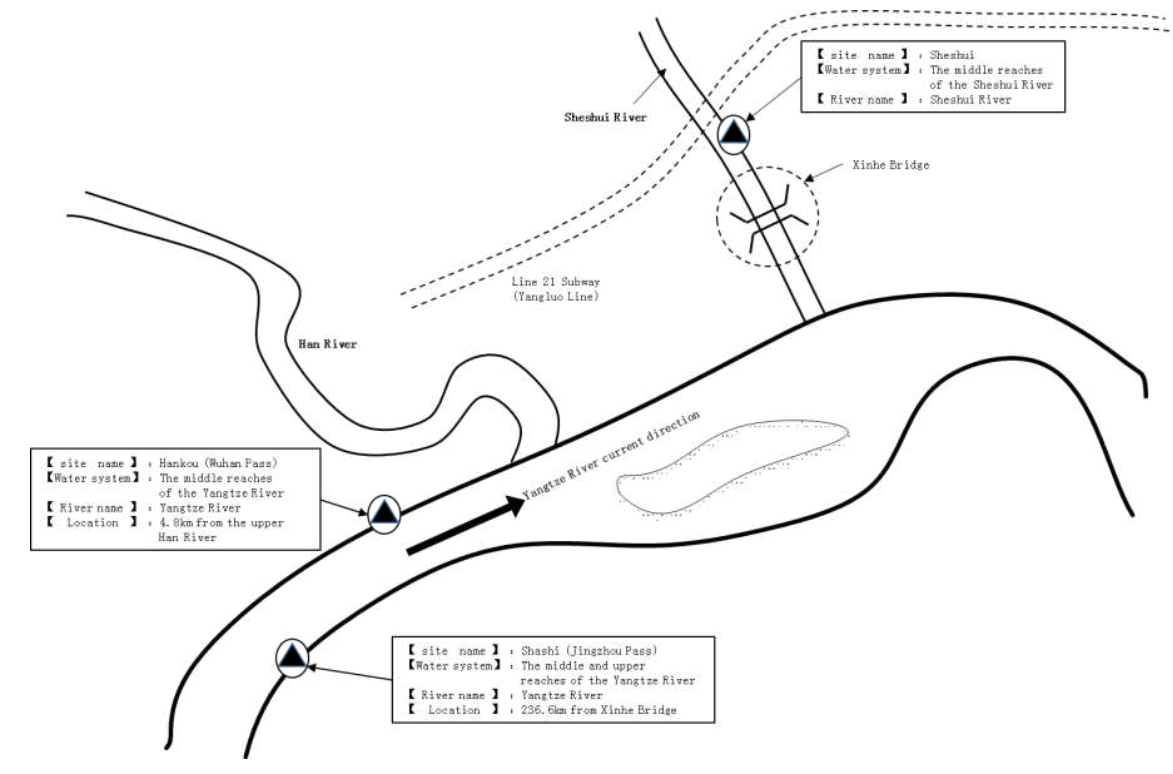

Fig. 3 Map showing the locations of Xinhe Bridge and the hydrological monitoring stations

\subsection{Data preprocessing}

Step1 Direct data acquisition

A total of 134 sets of water level are selected from the data recorded in 2016 and 2017. The water levels of Yangtze River $\left(\mathrm{X}_{1}\right)$ and Sheshui River $\left(\mathrm{X}_{3}\right)$, and their water level difference $\left(\mathrm{X}_{3}\right)$ are adopted from the water information table of the Hydrological Information Forecasting Office of the Hydrological and Water Resources Bureau of Hubei Province that records data once every hour. The data of 8 am each day that accurately match the meteorological data are selected and listed in Table 3.

Table 3 Selected raw data for modeling

\begin{tabular}{|c|c|c|c|c|c|}
\hline Number & Data & $\begin{array}{c}\text { Yangtze River } \\
\text { water level } X_{1} \quad(\mathrm{~m})\end{array}$ & $\begin{array}{c}\text { Water level difference } \\
\text { between Shashi and } \\
\text { Hankou } \mathrm{X}_{2}(\mathrm{~m})\end{array}$ & $\begin{array}{l}\text { Sheshui Rever } \\
\text { water level } X_{3} \\
\text { (m) }\end{array}$ & $\begin{array}{c}\text { Xinhe Bridge } \\
\text { Water Level (m) }\end{array}$ \\
\hline 1 & 2016.1.01 & 16.25 & 14.63 & 21.63 & 13.26 \\
\hline 2 & 2016.1.11 & 15.61 & 15.63 & 21.37 & 13.38 \\
\hline 3 & 2016.1.21 & 16.58 & 15.11 & 21.46 & 13.45 \\
\hline$\cdots$ & $\cdots$ & $\cdots$ & $\cdots$ & $\ldots$ & $\ldots$ \\
\hline 30 & 2016.7.05 & 27.48 & 13.41 & 27.25 & 25.27 \\
\hline
\end{tabular}




\begin{tabular}{cccccc}
\hline 31 & 2016.7 .07 & 28.36 & 12.27 & 28.33 & 24.99 \\
32 & 2016.7 .09 & 28.19 & 11.24 & 27.95 & 25.03 \\
$\ldots$ & $\ldots$ & $\ldots$ & $\ldots$ & $\ldots$ & $\ldots$ \\
67 & 2016.12 .31 & 14.91 & 15.96 & 21.42 & 14.52 \\
$\ldots$ & $\ldots$ & $\ldots$ & $\ldots$ & $\ldots$ & $\ldots$ \\
87 & 2017.06 .01 & 19.77 & 15.28 & 21.46 & 21.93 \\
88 & 2017.06 .06 & 20.1 & 13.93 & 21.29 & 22.34 \\
$\ldots$ & $\ldots$ & $\ldots$ & $\ldots$ & $\ldots$ & $\ldots$ \\
133 & 2017.12 .21 & 14.2 & 16.27 & 21.96 & 15.48 \\
134 & 2017.12 .31 & 13.78 & 17.02 & 22.27 & 14.32 \\
\hline
\end{tabular}

184

185

186

187

188

189

\section{0}

191

192

193

194

195

196

197

Step2 Meteorological data processing

The meteorological data are preprocessed by the method mentioned above. Based on the historical rainfall distribution of Wuhan city, the data of July and August when the precipitation is relatively concentrated are extracted. The daily weather conditions are scored, and the average value of weather changes of every 10 days is taken as the value of influencing variable X4. Fig. 4 shows the scores of the weather conditions in 2016 and 2017.
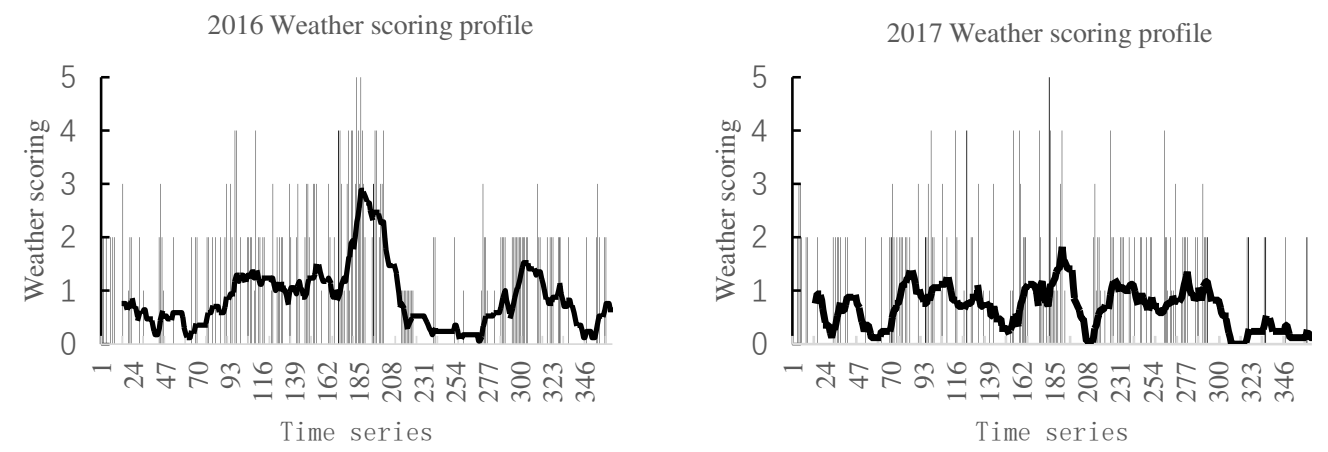

Fig. 4. Time series weather scoring

\subsection{PSO-SVM and SVM modeling}

The PSO-SVM modeling for the short-to-medium-term water level prediction of the Xinhe Bridge is conducted in the MatlabR2018a software and the results are compared with those of the conventional SVM modeling (Tan et al. 2019).

\section{Step1 PSO-SVM modeling}

From the 134 sets of data, 104 sets are randomly selected as the training dataset. The penalty factor $c$ and 
the function parameter $g$ of SVM are then automatically optimized by PSO to establish a PSO-SVM water level prediction model. The remaining 30 sets of data are used as the test dataset for the regression error analysis and accuracy evaluation. The initial values of the PSO algorithm are set as: number of particle swarms, 30; maximum number of iterations, 300; particle dimension, 2; and acceleration factors, $c_{1}=1.5$ and $c_{2}=1.7$. The search ranges of $c_{1}$ and $c_{2}$ are $[1,1000]$ and $[0.1,100]$, respectively. The inertia weight decreases linearly from 1.2 to 0.9 with the cycle number.

PSO gives the optimal penalty factor $c=241.6347$ and kernel parameter $g=0.2388$ that are fitted into the constructed model for water level prediction. The running time of POS-SVM model is $78.88 \mathrm{~s}$. The training time is $2.9 \mathrm{~s}$, and the prediction time is $0.09 \mathrm{~s}$.

Step2 SVM modeling

Similarly, 104 sets of data are randomly selected as the training dataset, and the remaining 30 sets of data are used as the test dataset. The maximum number of iterations is 500 and the maximum number of evolutions is 20 . The penalty factor $c=2.8284$ and the kernel parameter $g=1.4142$ are obtained after the cross-validation, which are then brought into the model for water level prediction.

\subsection{Comparative analysis of PSO-SVM and SVM prediction results}

Fig. 5 shows the MSE of fitness and the fitting curve of PSO-SVM model. Fig. 6 compares the PSOSVM and SVM prediction results and true values of the training data and Fig. 7 compares those of the test data. The relative errors between the PSO-SVM and SVM prediction results for the training dataset and the test dataset are shown in Fig. 8 and 9, respectively.

Fig. 5. Fitness curve of PSO-SVM water level prediction

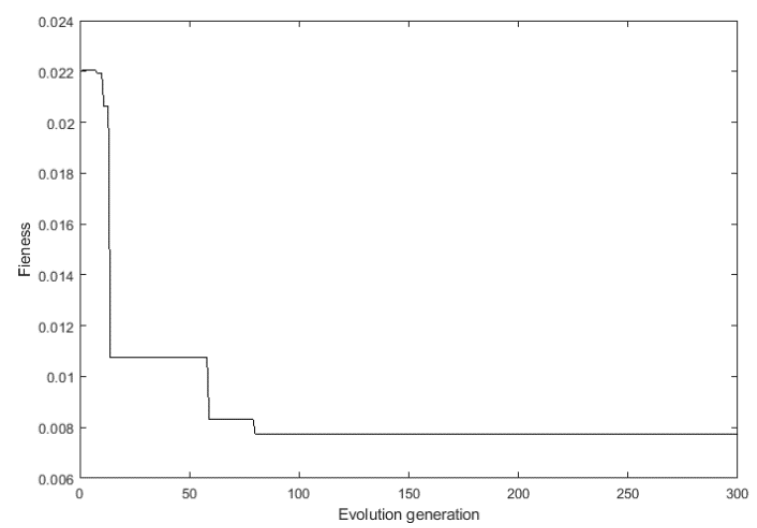


251

252

253

254

255

256
Fig. 8. Relative error between SVM and PSO-SVM prediction results of training dataset

Fig. 6. Comparison of PSO-SVM and SVM prediction results of training dataset with the true values

Fig. 7. Comparison of PSO-SVM and SVM prediction results of test set and the true values prediction results of training dataset
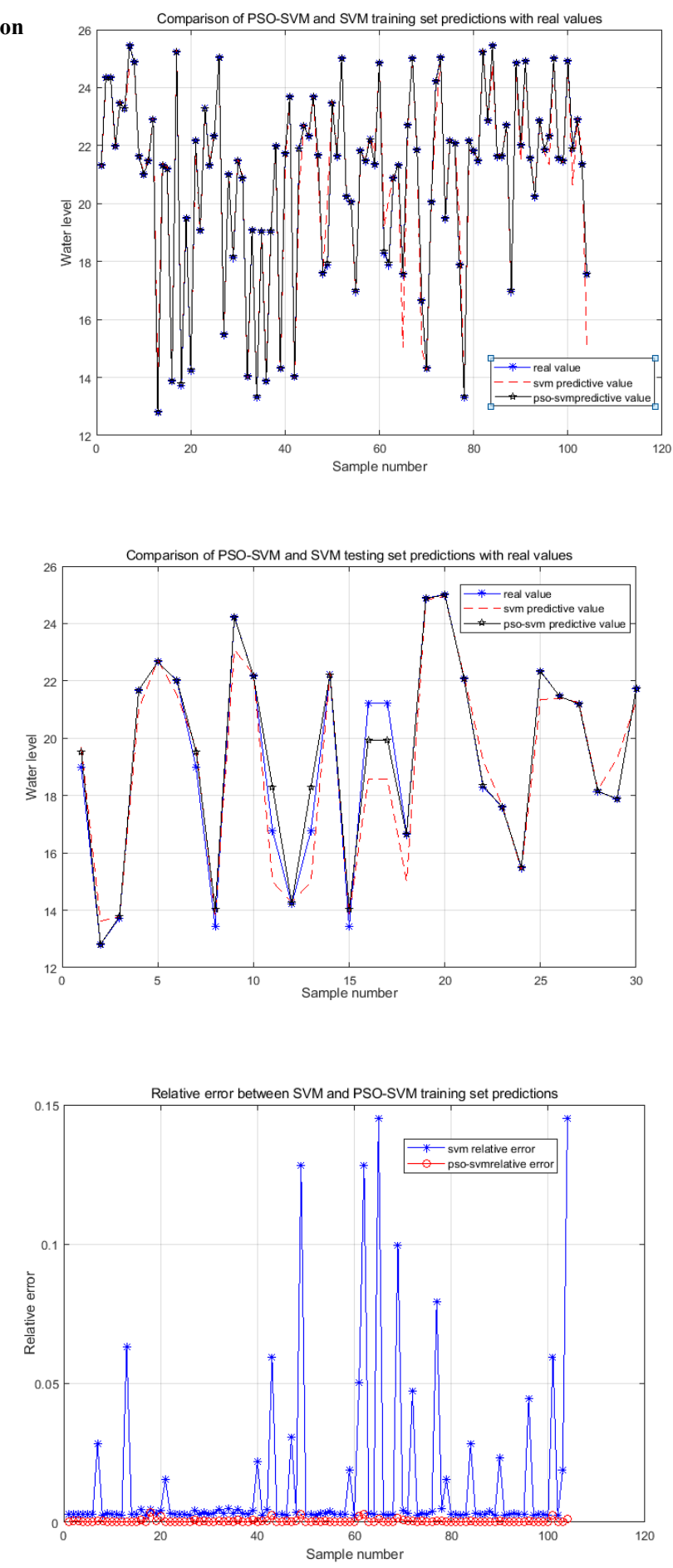
Fig. 9. Relative error between SVM and PSO-SVM prediction results of test dataset

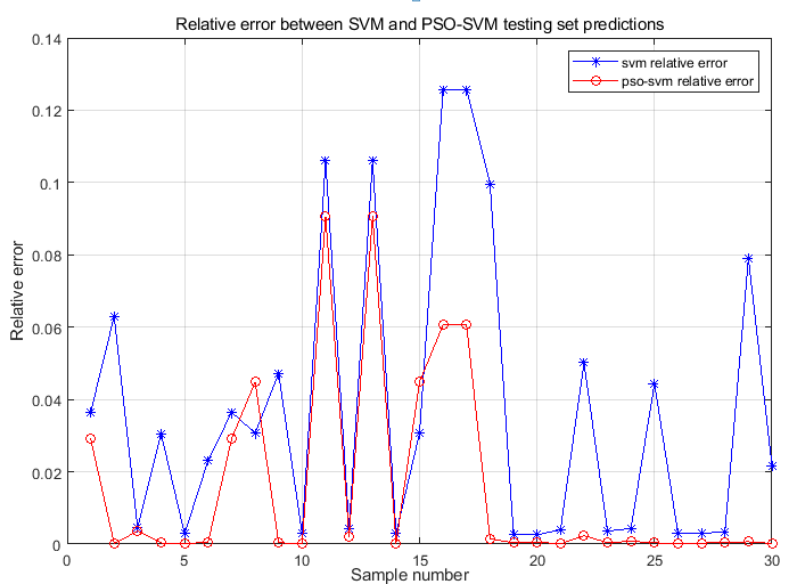

The PSO-SVM fitness curve suggests that the prediction value becomes almost stable in $\sim 70$ iterations. The relative error between the PSO-SVM prediction value and true value of the training dataset is much lower than that of SVM, indicating that the accuracy of PSO-SVM model is higher. The conclusion is further supported by the MSE and coefficient of determination of the predictions result as listed Table 4. It is clear that PSO-SVM model can predict the water level of small and medium-sized tributaries more accurately and reliably than SVM model, and thus is more suitable for the water level prediction of this type of rivers.

Table 3 Comparison of the prediction performances of PSO-SVM and SVM models

\begin{tabular}{ccc}
\hline Evaluation parameters & PSO-SVM & SVM \\
\hline MSE of training set & $5.108 \mathrm{e}-6$ & 0.009195 \\
$R^{2}$ of training set & 0.9999 & 0.96587 \\
MSE of sample set & 0.0077239 & 0.026154
\end{tabular}

$R^{2}$ of sample set

$0.98856 \quad 0.9237$

\section{Conclusions}

A water level predication model based on PSO and SVM algorithms has been constructed for the shortto-mid-term water level prediction of small and medium-sized rivers in urban using meteorological data as the variables. The accuracy and performance of the model are evaluated with real cases. The following conclusions are obtained.

Meteorological data, water level of adjacent water system, the difference between the water levels of two stations on the upstream of mainstream, and the historical water level of tributary are selected as the variables. There may be other factors affecting water level, which are not included in the models because of limited accessibility. Their identification and quantitative analysis are an undergoing project of our group.

The comparison of the PSO-SVM and SVM prediction suggests that the former can give smaller MSE 
and a higher coefficient of determination close to 1 . The $R^{2}$ of PSO-SVM prediction results of the training and test datasets are respectively 3.5\% and 7.02\% higher than those of SVM predictions. The high prediction accuracy of PSO-SVM model suggests that it is suitable for the short-to-medium term water level prediction.

The constructed model is successfully applied to the short-to-medium term water level prediction of a typical small- and medium-sized urban river. The model prediction can provide a scientific and accurate basis for the construction management of waterborne municipal projects and urban regional flood prevention. Future forecasts can be combined with hourly meteorological and water level data to achieve real-time prediction, improve water level prediction efficiency, and avoid the occurrence of flood disasters.

\section{Declarations}

\section{Funding}

The research was supported by General Project of the National Natural Science Foundation of China.

1. Large scale data driven spatiotemporal coupling modeling and risk perception for structural deformation safety of operating tunnels.(Award Number : 51778262)

2. Research on active control method of unsafe behavior of construction workers based on machine vision.(Award Number : 51978302)

\section{Conflicts of interest/Competing interests}

The authors declare that there is no conflict of interests regarding the publication of this article.

\section{Availability of data and material}

All data generated or analyzed during this study are included in this published article. [and its supplementary URL links]

\section{Code availability}

Not applicable

\section{Authors' contributions}

Yawei Qin: Conceptualization, Methodology, Supervision, Funding acquisition, Data curation. Yongjin Lei: Writing-original draft, Software, Modeling. Wanglai Ju: Software, Modeling, Data processing. Xiangyu Gong: Data collection, Supervision.

\section{References}

Data source links: http://113.57.190.228:8001/web/Report/RiverReport ; http://tianqi.2345.com/wea history/57494.htm

Adamowski J, Chan HF (2011) A wavelet neural network conjunction model for groundwater level forecasting Journal of Hydrology 407:28-40 
Anirudh V, Umes C Classification of rainy days using SVM. In: Proceedings of Symposium at HYDRO, Norfolk, Virginia, USA, 2007.

Barrameda KB, Lee SH, Kim S-Y Simulation of Flood Water Level Early Warning System Using Combination Forecasting Model. In: International Conference on Software Engineering Research, Management and Applications, 2018. Springer, pp 207-217

Caizhi S, Xueyu L (2003) Research on fuzzy Markov chain model with weights and its application in predicting the precipitation state Journal of Systems Engineering 18:294-299

Eslamian S, Gohari S, Biabanaki M, Malekian R (2008) Estimation of monthly pan evaporation using artificial neural networks and support vector machines J Appl Sci 8:3497-3502

Galavi H, Mirzaei M, Shul LT, Valizadeh N (2013) Klang River-level forecasting using ARIMA and ANFIS models Journal - American Water Works Association 105:E496-E506

Hin LZ, Othman Z (2020) Lake Chini Water Level Prediction Model using Classification Techniques. In: Computational Science and Technology. Springer, pp 215-226

Huang C-L, Dun J-F (2008) A distributed PSO-SVM hybrid system with feature selection and parameter optimization Applied soft computing 8:1381-1391

Khan MS, Coulibaly P (2006) Bayesian neural network for rainfall - runoff modeling Water Resources Research 42

Kia MB, Pirasteh S, Pradhan B, Mahmud AR, Sulaiman WNA, Moradi A (2012) An artificial neural network model for flood simulation using GIS: Johor River Basin, Malaysia Environmental Earth Sciences 67:251-264

Kisi O, Shiri J, Karimi S, Shamshirband S, Motamedi S, Petković D, Hashim R (2015) A survey of water level fluctuation predicting in Urmia Lake using support vector machine with firefly algorithm Applied Mathematics and Computation 270:731-743

Kühn M, Schöne T (2017) Multivariate regression model from water level and production rate time series for the geothermal reservoir Waiwera (New Zealand) Energy Procedia 125:571-579

Lee G, Lee S, Park H (2013) Improving applicability of neuro-genetic algorithm to predict short-term water level: a case study Journal of Hydroinformatics 16:218-230

Lin Z, Levy JK, Xu X, Zhao S, Hartmann J (2005) Weather and seasonal climate prediction for flood planning in the Yangtze River Basin Stochastic Environmental Research and Risk Assessment $19: 428-437$

Moghaddamnia A, Ghafari M, Piri J, Han D (2009) Evaporation estimation using support vector machines technique International Journal of Engineering and Applied Sciences 5:415-423

Nkiaka E, Nawaz N, Lovett J (2018) Effect of single and multi-site calibration techniques on hydrological model performance, parameter estimation and predictive uncertainty: a case study in the Logone catchment, Lake Chad basin Stochastic environmental research and risk assessment 32:16651682

Qiao W, Yang Z, Kang Z, Pan Z (2020) Short-term natural gas consumption prediction based on Volterra adaptive filter and improved whale optimization algorithm Engineering Applications of Artificial Intelligence 87:103323

Rao A, Upadhaya P, Pandey S, Poulose J (2019) Simulation of extreme water levels in response to tropical cyclones along the Indian coast: a climate change perspective Natural Hazards:1-22

Selakov A, Cvijetinović D, Milović L, Mellon S, Bekut D (2014) Hybrid PSO-SVM method for shortterm load forecasting during periods with significant temperature variations in city of Burbank 
Applied Soft Computing 16:80-88

Shiri J, Shamshirband S, Kisi O, Karimi S, Bateni SM, Nezhad SHH, Hashemi A (2016) Prediction of water-level in the Urmia Lake using the extreme learning machine approach Water resources management 30:5217-5229

Shrivastava NA, Khosravi A, Panigrahi BK (2015) Prediction interval estimation of electricity prices using PSO-tuned support vector machines IEEE Transactions on Industrial Informatics 11:322331

Simon B, Insa N, Lothar F Real-Time Water Level Prediction Based on Artificial Neural Networks. In: International Conference on Urban Drainage Modelling, 2018. Springer, pp 603-607

Tan X, Yu F, Zhao X (2019) Support vector machine algorithm for artificial intelligence optimization Cluster Computing 22:15015-15021

Wang J, Sui J, Guo L, Karney B, Jüpner R (2010) Forecast of water level and ice jam thickness using the back propagation neural network and support vector machine methods International Journal of Environmental Science \& Technology 7:215-224

Wei C-C (2012) Wavelet kernel support vector machines forecasting techniques: Case study on waterlevel predictions during typhoons Expert Systems with Applications 39:5189-5199

XIE Z-t, ZHANG X-f, TAN G-m, YANG F-1 (2005) Forecasting of stage-discharge relationship of Luoshan on the Yangtze River Journal of Wuhan University of Hydraulic and Electric Engineering:3

Yadav B, Eliza K (2017) A hybrid wavelet-support vector machine model for prediction of Lake water level fluctuations using hydro-meteorological data Measurement 103:294-301

YAO Z, XU J-p, KONG J-1, LIU S-b (2018) Prediction of River Water Level by GA-Elman Model Journal of Yangtze River Scientific Research Institute:9

Yarar A, Onucyıldız M, Copty NK (2009) Modelling level change in lakes using neuro-fuzzy and artificial neural networks Journal of Hydrology 365:329-334

Zakaria ZA, Shabri A (2012) Streamflow forecasting at ungaged sites using support vector machines Applied Mathematical Sciences 6:3003-3014

Zhang X, Guo X, Hu M (2016) Hydrological effect of typical low impact development approaches in a residential district Natural Hazards 80:389-400

Zhao G, Pang B, Xu Z, Xu L (2019) A Hybrid Machine Learning Framework for Real-Time Water Level Prediction in High Sediment Load Reaches Journal of Hydrology:124422

Zhu X, Lu C, Wang R, Bai J (2005) Artificial neural network model for flood water level forecasting Journal of Hydraulic Engineering 36:806-811 